\title{
ADESÃO AO TRATAMENTO ANTI-HIPERTENSIVO: UMA ANÁLISE CONCEITUAL ${ }^{1}$
}

\author{
ADHERENCE TO THE ANTIHYPERTENSIVE TREATMENT: A CONCEPTUAL ANALYSIS
}

ADHESIÓN AL TRATAMIENTO ANTIHIPERTENSIVO: UNA ANALISE CONCEPTUAL

\section{Gilmara Barboza da Silva Araújo², Telma Ribeiro Garcia ${ }^{3}$}

RESUMO: A hipertensão arterial sistêmica (HAS) ocupa lugar de destaque no contexto da transição epidemiológica, e constitui um dos principais fatores de risco para o aparecimento das doenças cardíacas. O controle da HAS está diretamente relacionado ao grau de adesão do paciente ao regime terapêutico. Este estudo objetiva analisar o conceito "Adesão ao tratamento anti-hipertensivo", identificando os possíveis fatores antecedentes, os atributos críticos e as conseqüências do fenômeno; e elaborar um modelo teórico que incorpore os achados da análise conceitual, ofereça subsídios para a construção de instrumentos de medida do conceito de "Adesão ao tratamento anti-hipertensivo" e sirva como base para o planejamento de atividades educativas direcionadas à clientela de hipertensos. O estudo seguiu a metodologia de análise conceitual proposta por Lorraine O. Walker e Kay C. Avant e foi realizado através de um levantamento bibliográfico de artigos científicos da área médica e de enfermagem pertinentes à temática, publicados, nos idiomas português e inglês, no período de janeiro/1995 a julho/2001. Como antecedentes da "Adesão ao tratamento anti-hipertensivo" foram identificados aspectos relacionados ao paciente, ao regime terapêutico e ao sistema de saúde. Dois atributos foram identificados para o conceito: a participação ativa no tratamento e a realização de mudanças no estilo de vida. Quanto às conseqüências, foram identificadas a pressão arterial controlada, a redução na incidência ou o retardamento na ocorrência de complicações e a melhoria da qualidade de vida do hipertenso.

PALAVRAS-CHAVE: Hipertensão; Pressão arterial; Conduta de saúde; Participação do paciente.

ABSTRACT: The arterial systemic hypertension occupies a prominent place in the epidemiological transition context and constitutes one of the main risk factors for the appearance of heart diseases. The control of hypertension is directly related to the degree of the patient's adherence to the therapeutic regime. This study aims to analyze the concept of "Adherence to the antihypertensive treatment", identifying the possible antecedent factors, the critical attributes and the consequences of the phenomenon; and also to elaborate a theoretical model which incorporates the findings of the conceptual analysis and offers subsidies for the construction of instruments to measure the concept, as well as a basis for educational activities planned to the patients with hypertension. The study has adopted the conceptual analysis methodology proposed by Lorraine $\mathrm{O}$. Walker and Kay C. Avant, and it was carried out through a bibliographical survey of scientific articles on the medical and nursing field relating to this matter, published in the Portuguese and English languages, from January 1995 to July 2001. Several aspects related to the patient, to the therapeutic regime and to the Health System were identified as antecedents of the "Adherence to the antihypertensive treatment". Two attributes were identified for the concept: the active participation in the treatment and the accomplishment of changes in the life style. The reduction in the incidence or the delay in the occurrence of complications, the controlled arterial pressure and the improvement of the quality of life of patients with hypertension were identified as consequences of the concept of "Adherence to the antihypertensive treatment".

KEYWORDS: Hypertension; Blood pressure; Health behavior; Participación del paciente.

RESUMEN: La hipertensión arterial sistemica ocupa un lugar prominente en el contexto de la transición epidemiológica y constituye uno de los factores de riesgo principales para enfermedades del corazón. El controlo de la hipertensión se relaciona directamente al grado de la adhesión del paciente al programa terapéutico. Este estudio objetiva analizar el concepto de "Adhesión al tratamiento antihipertensivo", identificando los posibles factores antecedentes, los atributos críticos y las consecuencias del fenómeno; y elaborar un modelo teórico que incorpore los hallazgos del análisis conceptual y ofrezca subsidios para la construcción de instrumentos de medida del concepto, así como una base teórica para la planificación de actividades educativas direccionada a

\footnotetext{
Extraído de ARAÚJO, G. B. da S. Adesão ao tratamento antihipertensivo: análise conceitual. 2002. 119p. Dissertação (Mestrado em Enfermagem) Centro de Ciências da Saúde, Universidade Federal da Paraíba, João Pessoa-PB.

2 Enfermeira. Mestre em Enfermagem pela UFPB, área de concentração Enfermagem de Saúde Pública. João Pessoa/PB.

3 Enfermeira. Doutora em Enfermagem pela EERP-USP. Professora (aposentada) do Departamento de Enfermagem de Saúde Pública e Psiquiatria, Centro de Ciências da Saúde, UFPB. Pesquisadora do CNPq. João Pessoa/PB
} 
ARAÚJO, G. B. S.; GARCIA, T. R. Adesão ao tratamento anti-hipertensivo: uma análise conceitual. Revista Eletrônica de Enfermagem, v. 08, n. 02, p. 259 - 272, 2006. Disponível em http://www.fen.ufg.br/revista/revista8 2/v8n2a11.htm

los pacientes con hipertensión. El estudio ha adoptado la metodología del análisis conceptual propuesta por Lorraine O. Walker y Kay C. Avant y fue realizado a través de un estudio bibliográfico de artículos científicos de las áreas de medicina e enfermería pertinentes a la temática, publicados en los idiomas portugués y ingles, en el periodo de enero de 1995 al julio de 2001. Como antecedentes de la "Adhesión al tratamiento antihipertensivo" se identificaron aspectos relacionados tanto al paciente como al programa terapéutico y al Sistema de Salud.

\section{INTRODUÇÃO}

A hipertensão arterial sistêmica (HAS) ocupa lugar de destaque no contexto da transição epidemiológica que vem ocorrendo no Brasil a partir da década de 1960 do século passado (ROUQUAYROL, 1994). Até o final dos anos 1940 pouco se conhecia sobre a epidemiologia da HAS e não havia critérios padronizados e amplamente divulgados para sua definição; a partir de 1970, começou o interesse pela HAS como problema de saúde pública, quando já eram claros os indícios de sua relação com óbitos por doenças cardiovasculares (LESSA, 1993).

As estimativas de prevalência de HAS no Brasil apresentam grande variação, em função dos diferentes critérios de classificação e instrumentos de medida utilizados. Para ROUQUAYROL (1994), a divergência observada na magnitude das prevalências pode ser influenciada pelos grupos e pelo tipo de população estudada. A despeito dessas diferenças de abordagens, estudos populacionais disponíveis estimaram prevalências entre 15 e $30 \%$ para os homens e entre 15 e $17 \%$ para as mulheres o que, sem dúvida, coloca a HAS como principal fator de risco para as doenças cardiovasculares (CHOR, 1998). Para que se tenha uma idéia da magnitude do problema, cerca de $85 \%$ dos pacientes que sofrem acidente vascular encefálico (AVE) e $40 \%$ das vítimas de infarto agudo do miocárdio (IAM) apresentam HAS associada (BRASIL, 2001).

Sendo a HAS uma doença crônica, ela pode ser controlada, mas não curada, requerendo tratamento por toda a vida. Um fato preocupante é que muitos indivíduos só descobrem que são portadores da doença quando apresentam complicações grave, haja vista que a HAS pode evoluir por um longo período sem ocasionar sintomas. BRANDÃO et al (1993) enfatizam que, de toda a população de hipertensos, cerca de um terço não sabe que tem a doença e, dentre os que sabem, apenas a metade adere, efetivamente, ao tratamento. Segundo a Sociedade Brasileira de Hipertensão (SBH, 2003), "estudo concluído em julho de 2003 pela OMS revelou que a manutenção do tratamento por parte dos pacientes portadores de doenças crônicas nos países desenvolvidos é realizada por apenas
Fueran identificados dos atributos para el concepto: la participación activa en el tratamiento y el logro de cambios en el estilo de vida. En cuanto a las consecuencias, fueran identificadas la presión arterial controlada, la reducción en la incidencia o el retardo en la ocurrencia de complicaciones y la mejora de la calidad de vida de pacientes con hipertensión.

PALABRAS CLAVE: Hipertensión; Presión sanguínea; Conducta de salud; Pacient Participation.

$50 \%$ dos doentes. Mas nos países em desenvolvimento esse número é muito menor."

A preocupação com a adesão às recomendações terapêuticas data de muito tempo. Autores como AKASHI et al (1998), CARVALHO FILHO \& CURIATI (1996) e COSTA (2001), entre outros, são unânimes ao pontuar os ganhos relacionados ao controle e tratamento da HAS. Segundo KUNCL \& NELSON (1997), KJELLGREN et al (1995) e LAHDENPERÄ \& KYNGÄS (2000), dentre os prejuízos resultantes da não adesão sobressai o inadequado controle da HAS; o aumento de complicações e de mortes resultantes dessas complicações; o aumento dos gastos com admissões hospitalares e o absenteísmo no trabalho. Para MARCON et al (1995), a adesão do paciente ao regime terapêutico é de suma importância para o controle dos sintomas e progressão da doença. SARQUIS et al (1998) enfatizam que a meta primordial das ações das equipes de saúde deve ser a de buscar otimizar a adesão do hipertenso ao tratamento.

De acordo com a Sociedade Brasileira de Hipertensão (SBH, 2003), estudo realizado pela OMS ressalta, como prejuízos do não-cumprimento do tratamento, as complicações médicas e psicossociais da enfermidade, a redução da qualidade de vida dos pacientes, a maior probabilidade de resistência aos fármacos e o desperdício dos recursos assistenciais.

Considerando-se a problemática envolvida nessa questão, como também o papel de destaque que ocupa no controle da doença, acredita-se ser de extrema relevância a análise do conceito "Adesão ao tratamento anti-hipertensivo", vez que permitirá uma compreensão mais abrangente do fenômeno, fornecendo a base para que se possa intervir de modo mais eficaz na tentativa de ajudar pacientes hipertensos a controlar ou a evitar danos causados por uma HAS não controlada. Desse modo, foram estabelecidos como objetivos para esse estudo: realizar a análise do conceito "Adesão ao tratamento anti-hipertensivo", identificando os possíveis fatores antecedentes (ou preditores), os atributos críticos e as conseqüências do fenômeno; e elaborar um modelo teórico que incorpore os achados da análise conceitual, ofereça subsídios para a construção de instrumentos de medida do conceito e sirva como 
ARAÚJO, G. B. S.; GARCIA, T. R. Adesão ao tratamento anti-hipertensivo: uma análise conceitual. Revista Eletrônica de Enfermagem, v. 08, n. 02, p. 259 - 272, 2006. Disponível em http://www.fen.ufg.br/revista/revista8 2/v8n2a11.htm

base para o planejamento de atividades educativas direcionadas à clientela de hipertensos.

\section{METODOLOGIA}

Realizou-se a análise do conceito "Adesão ao tratamento anti-hipertensivo", utilizando-se passos da metodologia proposta por WALKER \& AVANT (1995): seleção do conceito; determinação dos objetivos da análise conceitual; identificação dos possíveis usos do conceito; determinação dos atributos críticos ou essenciais e dos eventos antecedentes e conseqüências do conceito.

Depois de se haver selecionado o conceito a ser analisado e de se haver estabelecido os objetivos da análise conceitual, foi determinado que se verificaria como ele tem sido abordado em artigos publicados em periódicos da área da saúde. Para identificar os artigos a serem analisados foram acessadas as seguintes bases de dados, empregando-se os unitermos "hypertension", "hypertension treatment", "compliance" e "adherence", com os correlatos em português: Comprehensive Medline, que reúne as três maiores fontes bibliográficas da área biomédica, a saber, o Index Medicus, o Index of Dental Literature e o International Nursing Index; e o Lilacs, que reúne a literatura latinoamericana e do Caribe em ciências da saúde.

Além dessas duas bases de dados, utilizou-se o Sumário de Periódicos em Enfermagem, publicado pelo Serviço de Biblioteca e Documentação da Escola de Enfermagem da USP, que divulga o conteúdo dos títulos de periódicos recebidos, da área de Enfermagem e de áreas afins. Na busca de artigos focalizando a "Adesão ao tratamento antihipertensivo", foram pesquisados todos os volumes do Sumário de Periódicos em Enfermagem do período de 1995 a 2001.

Foram estabelecidos os seguintes critérios para a inclusão de artigos no estudo: a) focalizar o tema da pesquisa (HAS) e abordar o conceito a ser analisado; b) estar escrito nos idiomas português ou inglês; c) estar publicado, entre janeiro de 1995 e julho de 2001, em periódico indexado.

Inicialmente foram identificados 65 artigos nas fontes descritas. Após a aquisição, cada artigo foi submetido a leitura flutuante para apreciação do conteúdo e para confirmar se possuía elementos que serviriam de base para a análise conceitual pretendida. Decorrente desse screening, foram excluídos da amostra quinze artigos, dos quais treze não apresentavam elementos suficientes para realização da análise do conceito em questão; e dois por estarem escritos em idioma diferente dos preestabelecidos nos critérios de inclusão. Assim, a amostra do estudo ficou constituída por 50 artigos, dos quais 29 (58\%) em português e 21 (42\%) em inglês; 24 (48\%) escritos por profissionais da área de enfermagem, 24 (48\%) escritos por profissionais da área de medicina, e 2 (4\%) uma publicação conjunta de médicos e enfermeiras.

Realizou-se uma leitura criteriosa e objetivada dos 50 artigos que serviram de base para a análise do conceito, destacando-se, durante a leitura, os trechos que correspondiam aos elementos de interesse, ou seja, que se referiam a atributos críticos ou essenciais, a eventos antecedentes ou a conseqüências da "Adesão ao tratamento antihipertensivo". Procedeu-se, então, à digitação de todos os trechos dos artigos que foram destacados durante a leitura e, em seguida, os trechos digitados foram analisados individualmente, buscando-se, em um processo indutivo, identificar subcategorias e categorias de antecedentes, de atributos e de conseqüências do conceito estudado.

\section{ANTECEDENTES DA “ADESÃO AO TRATAMENTO ANTI-HIPERTENSIVO"}

\section{Relacionados ao paciente}

Os artigos analisados apontaram vários fatores antecedentes da "Adesão ao tratamento antihipertensivo" que se podia relacionar ao paciente, dentre os quais alguns diziam respeito a variáveis sociodemográficas, outros ao conhecimento e crenças do paciente sobre a HAS, e outros ao apoio familiar e social.

Com relação ao sexo, segundo alguns autores, as mulheres geralmente aderem mais ao tratamento, quando comparadas aos homens. No estudo de KYNGÄS \& LAHDENPERÄ (1999), enquanto 83\% das mulheres seguiam a dieta hipossódica, apenas $17 \%$ dos homens haviam aderido a essa recomendação. Resultados semelhantes foram encontrados no estudo realizado por MEDEL (1997), em que se verificou que um maior número de mulheres apresentaram alto nível de adesão, quando comparadas aos homens. SARQUIS et al (1998) também enfatizam que os homens tendem a ser menos aderentes que as mulheres, possivelmente porque elas demonstram uma maior preocupação com a saúde.

Os indivíduos de idade mais avançada são mais propensos à adesão. Em pesquisa realizada por SALA et al (1996), envolvendo pacientes hipertensos de um programa de unidade básica de saúde, verificou-se uma redução mais intensa da PA diastólica em indivíduos mais velhos. CHOR (1998), em estudo realizado com funcionários de um banco estatal no Rio de Janeiro, verificou que, enquanto $17,1 \%$ dos indivíduos mais jovens realizavam tratamento anti-hipertensivo, esse número aumentou para $77,4 \%$ em indivíduos de 45 anos ou mais. CAR et al (1991) explicam que o jovem não se sente vulnerável à doença, enquanto que o idoso, mais preocupado com a saúde, se apega ao tratamento como alternativa de prolongamento da vida.

Dois autores referiram que, quanto mais elevado o grau de instrução, maior também será o 
ARAÚJO, G. B. S.; GARCIA, T. R. Adesão ao tratamento anti-hipertensivo: uma análise conceitual. Revista Eletrônica de Enfermagem, v. 08, n. 02, p. 259 - 272, 2006. Disponível em http://www.fen.ufg.br/revista/revista8 2/v8n2a11.htm

nível de adesão. CHOR (1998) constatou que o número de indivíduos com curso superior que se tratava era seis vezes maior que o número de indivíduos que não tinha ingressado na universidade e realizava tratamento anti-hipertensivo. KYNGÄS \& LAHDENPERÄ (1999) verificaram que, nos pacientes hipertensos com maior nível de escolaridade, a adesão ao tratamento não medicamentoso era maior visto que, enquanto $46 \%$ dos hipertensos com formação de nível superior estavam dentro da faixa do peso normal, apenas $24 \%$ dos pacientes com nível educacional mais baixo estavam dentro dos padrões do peso recomendado. Para FREITAS et al (2001), as pessoas com um baixo nível educacional têm menos conhecimento de como prevenir as doenças e, em vista disso, menor nível de adesão.

Alguns autores mencionaram a situação financeira como fator diretamente relacionado à adesão. De acordo com CLARK et al (2000), o status econômico influencia na habilidade dos indivíduos em seguir as recomendações. No estudo de MEDEL (1997) foi verificado que os pacientes economicamente ativos eram mais aderentes ao tratamento do que os aposentados, atribuindo-se esse fato à questão econômica pois, ao se aposentar, os indivíduos sofrem perdas financeiras, que podem contribuir para o abandono do tratamento. SARQUIS et al (1998) resssaltam que a disponibilidade financeira deve ser um ponto importante a ser considerado no processo de adesão. Em trabalho realizado por TRENTINI et al (1996), os hipertensos relataram problemas decorrentes da dificuldade financeira, principalmente no tocante à compra de medicamentos e à compra de alimentos de acordo com as restrições dietéticas. Segundo CAR et al (1991) a situação sócio-econômica é um fator altamente interveniente na adesão, estando não só relacionada à compra de medicamentos, mas também a aspectos educacionais, culturais e sociais.

O estado civil foi outra variável sociodemográfica apontada como preditora da "Adesão ao tratamento anti-hipertensivo". No estudo de CHOR (1998), quando comparados aos solteiros, os hipertensos casados apresentaram chance duas vezes maior de realizar o tratamento; no de KYNGÄS \& LAHDENPERÄ (1999), enquanto $14 \%$ dos hipertensos casados fumavam, $34 \%$ dos solteiros ou divorciados não haviam conseguido abolir o vício.

$O$ conhecimento $e$ as crenças que os hipertensos têm sobre a doença constituem importantes antecedentes da "Adesão ao tratamento anti-hipertensivo", sendo fundamental nesse processo a percepção que os pacientes têm acerca da doença. MARCON et al (1995) afirmam que, para uma decisão específica de saúde ser tomada, é necessário que o indivíduo perceba a doença como ameaça. Em se tratando da HAS, essa questão é bastante problemática pois, sendo essa doença, na maioria das vezes, assintomática, os pacientes não a encaram como um problema de saúde que necessite de tratamento. Essa questão é abordada por SARQUIS et al (1998), para quem a ausência de sintomas contribui de forma marcante para a não adesão, ou para o abandono do tratamento. CHOR (1998) ressalta a dificuldade de convencer o paciente, muitas vezes assintomático, de que ele é doente e necessita de tratamento.

Em pesquisa realizada por CASTRO \& CAR (2000), comprovou-se que as modificações no estilo de vida relacionaram-se à presença de sintomatologia, à compreensão dos doentes sobre a doença e ao impacto desta em suas vidas. Para as autoras, o enfrentamento da cronicidade da HAS envolve, entre outros aspectos, a compreensão do seu significado, de acordo com as concepções do hipertenso sobre saúde-doença. No estudo realizado por KYNGÄS \& LAHDENPERÄ (1999), sintomas de pressão alta foram referidos por $61 \%$ das mulheres e $29 \%$ dos homens; dentre os hipertensos que referiram sintomas, $71 \%$ reduziram o sal da dieta, enquanto apenas $7 \%$ dos que não referiram sintomas seguiam a recomendação. SVENSSON et al (2000) verificaram que alguns dos pacientes que se declararam não aderentes acreditavam ser a HAS uma condição intermitente, que só precisava de tratamento na presença de sintomas.

De acordo com VIJAYALAKSMI et al (1997), o entendimento que o paciente tem de sua condição de saúde está diretamente relacionado a sua independência e cooperação com o regime terapêutico. HUNGERBUHLER et al (1995) verificaram que, pacientes com maior conhecimento sobre a doença e tratamento, apresentaram-se 3,6 vezes mais aderentes do que aqueles com um menor nível de conhecimento. Para JARDIM et al (1996), quando conhecem aspectos da doença, os pacientes se tornam elementos ativos no tratamento, ou seja, eles se tornam sujeitos e não simples objetos das ações a ele dirigidas. Para esses autores, os pacientes conscientes da importância do tratamento seguem mais corretamente as recomendações.

A vontade de conhecer os aspectos da doença e do tratamento também se constitui em um antecedente da "Adesão ao tratamento antihipertensivo", é o que colocam VIJAYALAKSMI et al (1997). Levando em consideração a importância do conhecimento do paciente no processo de adesão, o acesso a fontes de informação constitui ponto de destaque e, sem dúvida, também é considerado como antecedente do fenômeno.

A experiência anterior com a doença também é apontado como fator preditor da "Adesão ao tratamento anti-hipertensivo". Na pesquisa realizada por MARCON et al (1995), 34,1\% dos hipertensos entrevistados referiram, como motivo para iniciar o tratamento, a presença de alguém da família ou conhecido com alguma complicação relacionada à HAS não controlada.

A motivação para seguir as recomendações também foi outro fator apontado como evento 
ARAÚJO, G. B. S.; GARCIA, T. R. Adesão ao tratamento anti-hipertensivo: uma análise conceitual. Revista Eletrônica de Enfermagem, v. 08, n. 02, p. 259 - 272, 2006. Disponível em http://www.fen.ufg.br/revista/revista8 2/v8n2a11.htm

antecedente da adesão. O envolvimento no plano terapêutico constitui um dos fatores que podem motivar a realização do tratamento. MION JR. et al (1995) afirmam ser de extrema importância o envolvimento do hipertenso em seu autocuidado. Segundo CRUZ \& LIMA (1998), o autocuidado afeta diretamente a prevenção e o controle da pressão arterial.

A assiduidade dos pacientes aos encontros/consultas também ocupa lugar de destaque entre os preditores da adesão. JARDIM et al (1996) verificaram, em um programa de acompanhamento de hipertensos, que indivíduos mais assíduos aos encontros tiveram uma maior redução dos níveis tensionais. Segundo esses autores, a presença do paciente na unidade de saúde é determinante no controle da hipertensão, pois traz motivação individual e esta, por sua vez, leva a atitudes que contribuem para a redução da PA. Para CLARK et al (2000), encontros freqüentes propiciam uma melhor monitorização dos níveis pressóricos, assim como a oportunidade de ter mais acesso a informações que podem servir de base para a adesão.

O automonitoramento da pressão constitui um fator que pode aumentar a motivação individual e esta, por sua vez, pode conduzir à adesão. GRUENINGER (1995) destaca que, através do automonitoramento, o hipertenso, pela primeira vez, tem a oportunidade de acompanhar o comportamento de seu problema de saúde, o que proporciona uma autoresponsabilidade com efeito positivo na adesão. CLARK et al (2000) acrescentam que, para clientes não aderentes, uma pressão elevada detectada pelo automonitoramento pode motivar o retorno à adesão. Além da possibilidade de verificação da pressão pelo próprio hipertenso, após treinamento, também é importante que se estimule os pacientes ao automonitoramento, orientando-os a procurar regularmente os serviços de saúde para aferir a pressão arterial.

O apoio familiar e social também foi apontado como um evento antecedente da "Adesão ao tratamento anti-hipertensivo". Na visão de ARAÚJO et al (1998), a HAS provoca limitações no estilo de vida não somente do hipertenso, como também no estilo de vida dos outros elementos do núcleo familiar, pois a alteração na saúde de um dos membros da família acaba por provocar mudanças no todo, devendo-se incluir o grupo familiar no contexto do tratamento e acompanhamento dos hipertensos. CLARK et al (2000), enumerando as vantagens do trabalho realizado por um grupo de enfermeiras visitadoras na China, colocam que, ao visitar os pacientes em seu próprio ambiente, elas também entrariam em contato e desenvolveriam atividades educativas com a família e outras pessoas significativas, que são o suporte social dos pacientes. RUDD (1995) e FISCHMAN (1995) consideram que uma supervisão solidária por parte de pessoas significativas melhora o nível de adesão do paciente ao tratamento.
MEDEL (1997), em estudo realizado no Chile, verificou uma maior adesão ao tratamento e controle dos níveis tensionais nos pacientes que percebiam a família como apoio e suporte social. De acordo com a autora, as pessoas sendo melhor compreendidas dentro de seu contexto social são mais aderentes. No estudo realizado por CASTRO \& CAR (1999), a maioria dos hipertensos entrevistados referiram 0 apoio familiar como um fator facilitador da adesão. Segundo ARAÚJO et al (1998), para muitos hipertensos, um dos aspectos mais importantes da assistência é ter o apoio da família, o que pode ser exemplificado no comportamento do familiar de lembrar o hipertenso do horário das medicações e de orientá-lo na dieta, ou na disposição de algum dos membros da família para acompanhar o hipertenso às consultas pois, muitas vezes, em virtude de uma idade já avançada ou de outras limitações, o paciente não tem condições de se deslocar sozinho até o serviço de saúde.

\section{Relacionados à terapêutica}

Os artigos analisados evidenciaram fatores vinculados tanto à terapêutica farmacológica, como à não farmacológica, que favorecem a adesão do paciente ao tratamento.

No que se refere à terapêutica farmacológica, a simplificação é um dos pontos chave, enfatiza RUDD (1995). A diminuição do número de medicamentos utilizados e a diminuição da freqüência das dosagens, de preferência uma única dose diária, foram aspectos bastante citados nos artigos como fatores que levam à adesão. O horário da tomada dos medicamentos também foi apontado como fator que intervém na adesão, pois, correlacionando-se o horário das dosagens com atividades da rotina diária, que sirvam de lembretes, há melhora no nível de adesão. Por isso, autores como BITTAR (1995), PEPPER (1999), RUDD (1995) e KJELLGREN et al (1995) enfatizam a importância de uma prescrição medicamentosa individualizada, elaborada de acordo com o estilo de vida dos pacientes. MION JR. et al (1995) constataram que os pacientes preferem medicamentos administrados em dose única diária, com 0 horário de administração associado a atividades rotineiras matinais; e que eles preferem adotar formas que conciliem suas atividades com 0 tratamento proposto. Portanto, é de extrema importância levar em conta as necessidades e preferências pessoais por ocasião da realização da prescrição.

A eficácia em relação aos sinais e sintomas também aparece como fator preditor da adesão, assim como a pouca incidência de efeitos colaterais. Para KJELLGREN et al (1995), o tratamento farmacológico deve ser efetivo, ter poucos ou nenhum efeito colateral e não interferir negativamente na qualidade de vida dos pacientes. Segundo BITTAR (1995), com a introdução de novos agentes farmacológicos, a qualidade de vida dos hipertensos 
ARAÚJO, G. B. S.; GARCIA, T. R. Adesão ao tratamento anti-hipertensivo: uma análise conceitual. Revista Eletrônica de Enfermagem, v. 08, n. 02, p. 259 - 272, 2006. Disponível em http://www.fen.ufg.br/revista/revista8 2/v8n2a11.htm

tem melhorado significativamente e esse ponto parece estar influenciando diretamente na adesão. $\mathrm{O}$ autor ainda chama a atenção para a pouca ou nenhuma ocorrência de sintomas ligados à hipertensão, pois, quando os pacientes sentem intensos efeitos colaterais ao iniciar 0 uso de medicamentos anti-hipertensivos, podem fazer associação do tratamento com piora da qualidade de vida e, assim, aumenta a tendência a descontinuar o uso do medicamento. KUNCL \& NELSON (1997) acrescentam ser preciso reconhecer e responder às reações adversas antes que estas interfiram no tratamento. CARVALHO et al (1998) também ressaltam a importância da prescrição de acordo com a resposta do paciente. KYNGÄS \& LAHDENPERÄ (1999) verificaram que o maior nível de adesão estava relacionado à terapia farmacológica, sendo um dos motivos para a ocorrência desse fato os progressos dos medicamentos, a cada dia mais efetivos e com uma incidência cada vez menor de efeitos colaterais. Mas, apesar de todo o progresso relacionado às drogas anti-hipertensivas, a ocorrência de reações adversas muitas vezes é inevitável. O grau de tolerância dos pacientes a essas reações também tem importância na questão da adesão, explicam SARQUIS et al (1998). Desse modo, os pacientes precisam se esforçar no sentido de identificar e contornar as reações adversas das drogas, advertem KJELLGREN et al (1995).

O baixo custo dos medicamentos também foi mencionado nos artigos analisados como fator preditor de adesão. CASTRO \& CAR (1999) verificaram que mais da metade dos pacientes da amostra referiram dificuldade financeira para a compra do medicamento. GUERRA-RICCIO (2001) também obteve resultado semelhante, pois os problemas de ordem financeira foram apontados pelos participantes de seu estudo como um dos principais motivos para a descontinuação do tratamento farmacológico. FEITOSA (1996) refere que esse é um elemento de primordial importância na adesão a tratamentos de longa duração, como é o caso do tratamento anti-hipertensivo. Para AKASHI et al (1998) e PÓVOA (2001), o custo deve ser uma consideração importante na seleção do fármaco pois, sem dúvida, influencia na adesão ao tratamento.

No que se refere à terapêutica não farmacológica, CASTRO \& CAR (2000) e FREITAS et al (2001) consideram que o processo de aceitação e adaptação às modificações no estilo de vida relaciona-se diretamente ao seguimento do tratamento para o controle da HAS. Para CRUZ et al (1995), a experiência com um problema de saúde crônica exige a ativação de mecanismos de adaptação ao novo estilo de vida. Tal adaptação nem sempre é fácil, pois, muitas vezes, determina mudança de hábitos prazerosos, acrescenta CHOR (1998). CASTRO \& CAR (2000) verificaram que os pacientes relacionam 0 tratamento a mudanças problemáticas no cotidiano, como restrições alimentares, de lazer e trabalho. No estudo de KYNGÄS \& LAHDENPERÄ (1999), enquanto os mais altos níveis de adesão relacionavam-se à medicação, os piores estavam relacionados justamente ao tratamento não medicamentoso, incluindo a dieta, atividades físicas e ingestão de álcool. Segundo as autoras, os pacientes que utilizam medicamentos tendem a aderir menos às modificações no estilo de vida, pois acreditam que o uso das drogas é suficiente para se obter o controle da pressão arterial. Este comportamento é de fato preocupante, tendo em vista que os objetivos do tratamento não farmacológico são, além de reduzir as cifras tensionais, reduzir os fatores de risco cardiovasculares. Levando em conta esses aspectos, é recomendável que os profissionais de saúde discutam com os pacientes quais modificações estes consideram possíveis de serem realizadas, para que as práticas terapêuticas possam ser incorporadas efetiva e realisticamente. Para KJELLGREN et al (2000), é recomendável os profissionais pesquisem a opinião dos pacientes a respeito do tratamento medicamentoso e das mudanças no estilo de vida, como também de sua prontidão e capacidade de seguir o tratamento.

\section{Relacionados ao sistema de saúde}

Com relação aos antecedentes da "Adesão ao tratamento anti-hipertensivo" relacionados ao sistema de saúde, inúmeros autores pontuam a extrema relevância do acesso aos serviços. SARQUIS et al (1998) colocam que as políticas de saúde vigentes, a facilidade de acesso do paciente aos serviços de saúde e a qualidade do trabalho desenvolvido nesses serviços influenciam diretamente no processo de adesão. Essa questão também é abordada por COSTA (1998), para quem a maioria dos hipertensos, além de outros aspectos, não consegue acompanhar um tratamento ao longo do tempo, em virtude da dificuldade de acesso a um sistema público que facilite seu atendimento, a par do fato que a situação financeira da maioria dos doentes os impossibilta de procurar assistência em serviços privados. Levando isso em consideração, a ação integrada dos órgãos governamentais, das sociedades científicas médicas e demais associações da sociedade civil devem se direcionar no sentido de proporcionar um esquema de atenção que seja efetivo e de baixo custo (AKASHI et al, 1998), e que priorize ações e estratégias mínimas para o controle da hipertensão, tais como diagnóstico de casos, cadastramento dos portadores, busca ativa de casos, tratamento dos casos, diagnóstico precoce de complicações, atendimento de urgências e medidas preventivas (BRASIL, 2001).

A disponibilidade de medicamentos nos serviços para fornecimento aos pacientes também foi apontado como evento antecedente da "Adesão ao tratamento anti-hipertensivo". No estudo realizado por CASTRO \& CAR (1999), os hipertensos entrevistados mencionaram, como fatores facilitadores da adesão 
ARAÚJO, G. B. S.; GARCIA, T. R. Adesão ao tratamento anti-hipertensivo: uma análise conceitual. Revista Eletrônica de Enfermagem, v. 08, n. 02, p. 259 - 272, 2006. Disponível em http://www.fen.ufg.br/revista/revista8 2/v8n2a11.htm

ao tratamento, o recebimento gratuito de medicamentos, a facilidade de marcar consultas, a proximidade do serviço, como também o recebimento de vale transporte para o comparecimento às consultas. Segundo MARCON et al (1995), a facilidade de acesso ao serviço e o tratamento gratuito, incluindo consultas e exames, são fatores que, de fato, influenciam no seguimento do tratamento.

A disponibilidade de serviços de referência para encaminhamento de casos complexos, ou em caso de urgência, como a crise hipertensiva, é enfatizado por CLARK et al (2000). Outro fator relacionado à estrutura dos serviços de saúde e mencionado como evento antecedente da "Adesão ao tratamento anti-hipertensivo" é a disponibilidade de uma equipe multidisciplinar para o atendimento dos hipertensos. Sendo a HAS uma doença multicausal e multifatorial, exige diferentes abordagens, e só uma equipe multidisciplinar pode proporcionar essa ação diferenciada. JARDIM et al (1996) ressaltam que o atendimento dos hipertensos por profissionais de diferentes áreas melhora, em muito, a adesão à terapêutica recomendada. CAMPOS (1996) também pontua os benefícios da instituição da equipe multidisciplinar nos serviços, pois sua existência permite que os diversos fatores envolvidos na HAS e seu tratamento sejam examinados de modo mais profundo, visto que são abordados conjuntamente. A equipe multidisciplinar propiciará aos pacientes e à comunidade uma gama maior de informações, ajudando na adoção de atitudes efetivas e definitivas para o controle da hipertensão. Poderão fazer parte dessa equipe médicos, enfermeiros, nutricionistas, psicólogos, assistentes sociais, professores de educação física, farmacêuticos, funcionários administrativos, cada serviço adequando a composição da equipe de acordo com suas possibilidades e com a necessidade da clientela atendida. JARDIM et al (1996) enfatizam que deve haver harmonia de objetivos e uniformização de linguagem, além de um treinamento adequado dos membros da equipe.

No que se refere ao processo de atendimento, são citados nos artigos analisados vários eventos antecedentes da "Adesão ao tratamento antihipertensivo". Com relação à busca ativa de hipertensos, alguns autores pontuam sua importância pois subtende-se que, para aderir, o paciente necessita antes ter a sua doença diagnosticada. ALAVARCE et al (2000) enfatizam a necessidade de realização de campanhas direcionadas à população, informando a importância da verificação periódica da pressão arterial. MARCON et al (1995) também pontuam a relevância dessas campanhas, afirmando que os óbitos por doenças ligadas à hipertensão tenderão a diminuir quando a detecção e controle dessa doença forem práticas constantes nos serviços de saúde.
Pesquisa realizada em um serviço público de São Paulo por ALAVARCE et al (2000) constatou que em apenas 39\% das consultas foi realizada a aferição da pressão arterial. Levando esse fato em consideração, e tendo em vista a alta prevalência da HAS no nosso meio, esses autores destacam a importância da medida dos níveis tensionais para detecção precoce da doença. Sugerem, ainda, que essa aferição seja realizada em todas as consultas médicas, independentemente da especialidade, pois trata-se de um procedimento simples, fácil de ser executado e, se realizada corretamente, uma das maneiras mais rápidas, seguras e eficazes de se detectar a doença.

A organização do serviço, contemplando um sistema de controle da clientela registrada, de modo a identificar e buscar os faltosos, também é mencionada como fator preditor de adesão. De acordo com CAMPOS (1996), o contato com o paciente faltoso é um dos fatores que aumentam no paciente 0 sentimento de estar sendo cuidado, valorizado e estimulado. JARDIM et al (1996) chamam a atenção para a organização do serviço nesse sentido, sugerindo que o controle de retorno, assim como o contato e a busca de faltosos podem ser feitos por aerograma, contato telefônico ou por visita domiciliar, dependendo da situação. A instituição de um calling system é mencionada nos artigos analisados. Para BITTAR (1995) e GRUENINGER (1995), a existência no serviço de um programa de acompanhamento e um sistema de rastreamento de faltosos são fatores relacionados ao atendimento que concorrem para a adesão.

A freqüência das consultas e dos encontros hipertenso/profissional de saúde foi outro fator apontado como evento antecedente da adesão ao tratamento. No estudo de SALA et al (1996), foi observado que a redução da pressão arterial ocorreu sobretudo nos primeiros encontros, cabendo às consultas subseqüentes a função principal de manutenção dos níveis pressóricos já reduzidos. GUERRA-RICCIO (2001) constatou uma queda maior nos níveis pressóricos no grupo de pacientes com visitas de acompanhamento a cada 15 dias, quando comparados a outro grupo, cujos encontros aconteciam a cada 90 dias. Além disso, no grupo onde os encontros eram freqüentes, houve a manutenção da redução dos níveis tensionais ao longo do tratamento, o que demonstra 0 efeito favorável das visitas freqüentes para garantir o comportamento de adesão e manter a eficácia da terapêutica. De acordo com GUERRA-RICCIO (2001), um dos principais benefícios do número maior de visitas é a possibilidade de ajustes terapêuticos, no caso da ocorrência de efeitos colaterais; as visitas freqüentes também proporcionam uma mudança mais efetiva no estilo de vida e bem-estar aos pacientes, com possível redução da ansiedade e do estresse.

A satisfação do hipertenso com o atendimento é item fundamental para se conseguir bons níveis de 
ARAÚJO, G. B. S.; GARCIA, T. R. Adesão ao tratamento anti-hipertensivo: uma análise conceitual. Revista Eletrônica de Enfermagem, v. 08, n. 02, p. 259 - 272, 2006. Disponível em http://www.fen.ufg.br/revista/revista8 2/v8n2a11.htm

adesão e controle da HAS. Levando isso em consideração, a qualidade do trabalho desenvolvido nos serviços de saúde é de fundamental importância (SARQUIS et al, 1998). MEDEL (1997) ressalta que pacientes hipertensos não precisam apenas de cuidados físicos; necessitam, acima de tudo, de estímulo, esperança e compreensão. Segundo essa autora, a qualidade da relação que a equipe de saúde estabelece com o paciente é de fundamental importância, visto que o hipertenso tem necessidade de transmitir suas inquietudes, sintomas e limitações, além de apoio e reforço para conseguir adaptar-se à doença.

Atualmente é consenso geral que, além da consulta médica, se faz necessário oferecer ao paciente outros tipos de abordagens que, certamente, irão contribuir para melhorar a adesão. CADE (1999) constatou que a receptividade era um dos principais motivadores para que os pacientes freqüentassem um programa de controle da HAS, visto que, nesse programa, eles encontravam espaço para verbalizar sintomas, ansiedades, frustrações e dificuldades vividas no cotidiano em relação à doença e tratamento. Segundo CAMPOS (1996), para que os pacientes se sintam acolhidos, protegidos e estimulados a aderir, a equipe deve se constituir em suporte social adequado, vendo-os como pessoas, dotadas de história e circunstâncias de vida singulares, que precisam ser investigadas e trabalhadas.

O trabalho em grupos, com a participação de familiares dos hipertensos, tem se mostrado benéfico, concorrendo para melhorar a adesão. O grupo estimula a reflexão, amplia o nível de informação, e permite que cada um fale de si, havendo então a troca de experiências. Essa técnica funciona como suporte social na medida em que os pacientes estão reunidos em torno de um problema comum e sustentados por uma equipe que os apóia (CAMPOS, 1996). Bons resultados também foram conseguidos por CLARK et al (2000) em pesquisa em que um grupo de enfermeiras comunitárias realizava visitas periódicas aos hipertensos, durante as quais eram aferidos os níveis tensionais e também eram fornecidas informações. Na opinião das autoras, o ponto mais positivo era que as enfermeiras podiam conhecer e avaliar os pacientes inseridos dentro do seu próprio contexto social e familiar e, a partir daí, adequar o tratamento.

Boa parte dos autores dos artigos analisados atribui lugar de destaque ao relacionamento profissional de saúde/paciente como fator que exerce influência sobre a adesão do paciente ao tratamento. Segundo SVENSSON et al (2000), para otimizar o tratamento anti-hipertensivo, é importante formar uma aliança terapêutica entre o profissional e o paciente, de modo que as dúvidas e as dificuldades possam ser detectadas e resolvidas. KJELLGREN et al (1995) enfatizam a necessidade de uma relação de cooperação e colaboração, mantida em ambiente aberto ao diálogo. GRUENINGER (1995) ressalta a importância do apoio mútuo, em cujo âmbito o profissional é visto como um conselheiro e facilitador, e o paciente, como um parceiro, com a interação entre ambos voltada para a solução do problema. No âmbito dessa relação, segundo SVENSSON et al (2000), WILLIS (2000) e CLARK et al (2000), é essencial que haja menos autoritarismo por parte do profissional, sendo ideal o equilíbrio de poder entre ambas as partes.

Outros pontos relativos à conduta profissional são colocados pelos autores como preditores de adesão. De acordo com CURY JR. (1996), é preciso que o profissional esteja atento para a problemática da não adesão e, para KJELLGREN et al (1995), é de extrema importância que se discuta com os pacientes essa questão. Esses autores ainda criticam a posição adotada por alguns profissionais que, simplesmente, ignoram o fenômeno da não adesão, agindo como se todas as recomendações terapêuticas fossem seguidas sem problemas pelos pacientes. Levando isso em consideração, o monitoramento do processo de adesão tem seu grau de importância. Outra questão fundamental é que os profissionais encorajem a participação ativa do paciente no esquema terapêutico, promovendo o aumento da responsabilidade dos pacientes em relação a sua saúde (LAHDENPERÄ \& KYNGÄS, 2000).

Outros autores mencionam a importância de se conhecer o perfil dos pacientes, para que se possa elaborar um plano terapêutico individualizado. $O$ saber técnico para elucidar dúvidas também exerce papel de destaque, é o que comentam TRENTINI et al (1996). De qualquer forma, acrescenta RUDD (1995), nenhuma intervenção por si só é eficaz, sendo necessário uma combinação de medidas para que se possa atingir bons resultados.

No tocante à realização de ações educativas, inúmeros artigos as apontaram como fator primordial para a adesão dos pacientes ao tratamento. De acordo com GRUENINGER (1995) as ações educativas têm se tornado o novo paradigma dos cuidados primários em saúde e têm o objetivo principal de estimular mudanças de comportamento que sejam benéficas para a saúde; da mesma sorte, as ações educativas aumentam as habilidades dos pacientes para tomar decisões e para adaptar-se a uma condição de saúde específica.

$O$ efeito benéfico das ações educativas tem sido comprovado em algumas pesquisas. É o caso do estudo realizado por WANG \& ABBOTT (1998), em que os hipertensos conseguiram reduzir as cifras tensionais e a taxa de glicemia um ano após a instituição de medidas educativas, a exemplo de orientações sobre exercícios, dieta, importância do tratamento, entre outros aspectos da doença e do plano terapêutico. CAR et al (1991) também comprovaram os benefícios das ações educativas, pois a implementação de tais ações atuou satisfatoriamente no controle da HAS dos hipertensos 
ARAÚJO, G. B. S.; GARCIA, T. R. Adesão ao tratamento anti-hipertensivo: uma análise conceitual. Revista Eletrônica de Enfermagem, v. 08, n. 02, p. 259 - 272, 2006. Disponível em http://www.fen.ufg.br/revista/revista8 2/v8n2a11.htm

que participaram do estudo. As autoras consideram que, a partir do momento em que os indivíduos conhecem mais sobre a doença, sintomatologia e riscos do não tratamento, eles são levados a uma melhor aceitação da sua condição e da necessidade do tratamento.

DEVINE \& REIFSCHNEIDER (1995) alertam que, apesar da educação ter um largo efeito no conhecimento e, conseqüentemente, na adesão, esse efeito tende a diminuir com o tempo, fazendo-se necessário que tais medidas sejam efetivadas com certa periodicidade. Alguns autores referem vantagens das ações educativas grupais. Segundo MEDEL (1997), este tipo de abordagem é mais efetiva do que a individual, pois é mais variada e estimulante para os pacientes, que se encontram sem o estresse próprio da consulta. As ações educativas em grupo também fazem com que os integrantes percebam problemas comuns, sendo estimulados a desenvolver o autocuidado, aumentando assim a adesão e a eficácia do tratamento (MOREIRA et al, 1999). Os benefícios das ações educativas grupais foram evidenciados no estudo de TRENTINI et al (1996), em que destacam a importância de se utilizar uma estratégia que permita liberdade para refletir e criticar a realidade, permitindo que seja desenvolvida nos participantes a consciência da cidadania.

GRUENINGER (1995) informa que o conteúdo das informações deve ser completo, relevante e ajustado a necessidades percebidas pelos hipertensos, devendo abordar os fatos básicos sobre a HAS, origem e opções terapêuticas, sendo recomendável a utilização de materiais escritos e audiovisuais. Instruções claras, precisas e escritas em linguagem simples são destacadas por autores como KAPLAN (1995) e BITTAR (1995).

De modo geral, os artigos analisados mencionaram o efeito positivo das ações educativas sobre a adesão dos hipertensos ao tratamento, mas é válido lembrar que a instituição dessas medidas representa apenas um dos pontos dentro da imensa e complexa trama de fatores antecedentes identificados, sendo necessária, como já se mencionou, a associação de outras medidas específicas que possibilitem melhores níveis de adesão dos pacientes ao tratamento anti-hipertensivo.

\section{ATRIBUTOS DA "ADESÃO AO TRATAMENTO ANTI-HIPERTENSIVO"}

Cumprindo passos da metodologia para análise conceitual proposta por WALKER \& AVANT (1995), buscou-se determinar que atributos críticos ou essenciais do conceito "Adesão ao tratamento antihipertensivo" eram descritos pelos autores dos artigos analisados.

A participação ativa no tratamento foi destacada nos artigos como um importante atributo do conceito. KJELLGREN et al (1995) caracterizam o paciente aderente como uma pessoa ativa, que tem expectativas em relação aos profissionais que prestam os cuidados e é capaz de tomar decisões próprias relativas à sua saúde. KYNGÄS \& LAHDENPERÄ (1999) também ressaltam a participação ativa do paciente como um importante atributo do conceito. Segundo esses autores, intenção, responsabilidade e colaboração fazem parte dessa participação ativa. SMELTZER \& BARE (1998) explicam que, ao aderir ao tratamento, o paciente assume um papel mais ativo, auto determinando e alterando seus hábitos de vida. Nessa ótica, adesão é reconceitualizada, de uma atividade linear e controladora, para um esforço participativo incluindo profissional e paciente. Assim, segundo VIVIAN (1996), é esperado que pacientes aderentes participem das decisões e também assumam a responsabilidade por sua saúde e, por isso, propõe um modelo de adesão participativo e colaborativo, com uma divisão da responsabilidade dos cuidados entre profissionais e pacientes.

O uso correto dos medicamentos também se constitui importante característica dos pacientes que aderem ao tratamento, afirmam JARDIM et al (1996), MARCON et al (1995), entre outros autores. Vale salientar que a realização do tratamento medicamentoso é um dos pontos mais abordados nos estudos que tratam da adesão, sendo um dos itens freqüentemente avaliados.

KJELLGREN et al (1995) mencionam o monitoramento do tratamento como atributo da adesão. Entende-se essa característica como sendo a responsabilidade em cuidar-se, ou seja, o hipertenso percebe que o sucesso do tratamento depende não apenas do profissional de saúde, mas também dele próprio, passando assim a se preocupar em atingir o controle dos níveis pressóricos. O comparecimento pontual aos encontros ou consultas agendadas também aparece nos artigos analisados como sendo um atributo da adesão ao tratamento, sendo ressaltado por JARDIM et al (1996) e MARCON et al (1995), entre outros autores.

Outro atributo considerado relevante para 0 conceito "Adesão ao tratamento anti-hipertensivo" foi a realização de mudanças no estilo de vida, incluindo o seguimento do regime dietético prescrito, a prática regular de exercícios físicos e o monitoramento do estresse. Segundo TRENTINI et al (1996), alimentação adequada, exercícios físicos e, principalmente, ausência de angústia caracterizam um estilo de vida que pode ser considerado como saudável. CURY JR. (1996) e CAMPOS (1996) consideram que, apesar da intensidade das situações ambientais estressantes ter influência na elevação da pressão arterial, se o hipertenso adotar um melhor posicionamento frente a elas, o efeito dos fatores que as desencadeiam fica atenuado.

Em síntese, a participação ativa no tratamento, incluindo 0 uso correto dos medicamentos, o monitoramento do tratamento e o comparecimento pontual aos encontros, assim como as modificações 
no estilo de vida no que se refere ao seguimento do regime dietético, a práticas regulares de exercícios físicos e ao monitoramento do estresse, foram evidenciados nos artigos com atributos do conceito adesão ao tratamento anti-hipertensivo.

Para WALKER \& AVANT (1995), os atributos dos conceitos não são imutáveis. Atualmente, pelos resultados obtidos neste estudo, não se pode restringir o conceito "Adesão ao tratamento antihipertensivo" ao mero cumprimento das recomendações médicas, como há alguns anos era definido e como alguns ainda o definem. Hoje, sem dúvida, a participação ativa do paciente no tratamento se constitui o principal atributo desse conceito.

\section{CONSEQÜÊNCIAS DA "ADESÃO TRATAMENTO ANTI-HIPERTENSIVO"}

AO

Nos artigos analisados foram identificadas três conseqüências do conceito "Adesão ao tratamento anti-hipertensivo" - o controle dos níveis tensionais; a redução na incidência ou retardamento na ocorrência de complicações e a melhoria da qualidade de vida do hipertenso.

Para MOREIRA et al (1999) e CASTRO \& CAR (1999) a adesão do cliente ao tratamento é fator fundamental para o controle dos níveis tensionais. Desta forma, a principal conseqüência da adesão ao tratamento é uma pressão sangüínea controlada, como afirmam LAHDENPERÄ \& KYNGÄS (2000), BITTAR (1995), WANG \& ABBOTT (1998), entre outros autores. A partir do controle dos níveis tensionais, outros eventos são citados como conseqüências da adesão, como a redução na incidência ou retardamento na ocorrência de complicações. CHOR (1998) exemplifica essa questão citando o caso dos Estados Unidos que, nos últimos vinte anos, obtiveram uma redução de $60 \%$ na mortalidade por doenças cardiovasculares e de $53 \%$ na mortalidade por doenças coronarianas. Segundo a autora, essas reduções ocorreram em virtude do aumento do número de hipertensos diagnosticados, que realizam 0 tratamento $e$ conseguem controlar os níveis tensionais.

No processo de análise realizado, percebeu-se que as conseqüências estão intimamente relacionadas, ou seja, a adesão promove o controle dos níveis pressóricos; esse controle, por sua vez, provoca a redução na incidência ou retardamento na ocorrência de complicações e todos esses eventos, sem dúvida, levam à melhoria da qualidade de vida do hipertenso. KYNGÄS \& LAHDENPERÄ (1999) expressam de forma clara essa interligação quando afirmam que os principais objetivos do controle dos níveis pressóricos são a melhoria da qualidade de vida, a prevenção de complicações e, com isso, a diminuição da morbi-mortalidade. Assim, a promoção da adesão é essencial para que os hipertensos alcancem esses objetivos.

\section{MODELO TEÓRICO DO PROCESSO DE "ADESÃO AO TRATAMENTO ANTI-HIPERTENSIVO"}

Como resultado do estudo realizado, foi elaborado um modelo teórico (Fig. 1) englobando os três elementos envolvidos na análise do conceito "Adesão ao tratamento anti-hipertensivo".

Figura 1 - Modelo teórico do processo de "Adesão ao tratamento anti-hipertensivo". 


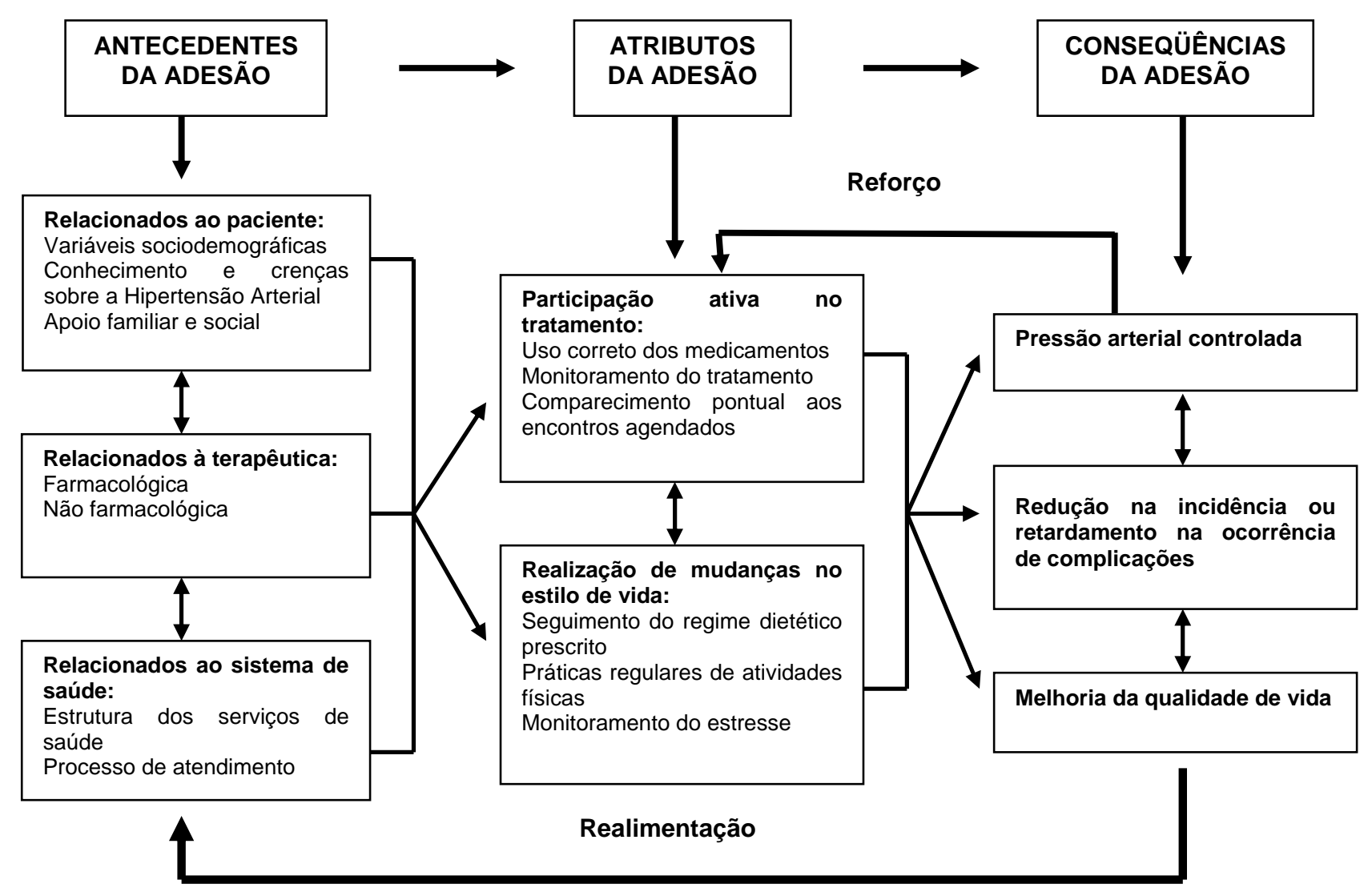

Levando em consideração os achados do estudo, compreende-se a "Adesão ao tratamento antihipertensivo" como um fenômeno que se evidencia pela participação ativa do paciente no plano terapêutico, considerada o atributo crítico do conceito, uma vez que o paciente não se constitui em um mero cumpridor de recomendações médicas; ao contrário, é visto como sujeito do processo, ou seja, como um ser que toma decisões e assume, juntamente com os profissionais que o assistem, a responsabilidade pelo tratamento.

Através da participação ativa, o portador de hipertensão compartilha a responsabilidade pelo sucesso da terapêutica farmacológica e não farmacológica, ou seja, cumpre os pontos do regime de tratamento no que diz respeito ao uso correto dos medicamentos; comparece aos encontros agendados e monitora o plano terapêutico e seus resultados. Além disso, realiza modificações no estilo de vida, adotando efetivamente o regime dietético prescrito, praticando regularmente atividades físicas $\mathrm{e}$ monitorando o estresse.

A análise realizada permitiu identificar uma evolução no significado do conceito "Adesão ao tratamento anti-hipertensivo" que, atualmente, não se limita ao mero cumprimento das recomendações médicas, mas demanda a participação ativa do paciente no tratamento. Considera-se que, ao se tornar participante ativo do processo, o paciente aumenta as chances de incorporar em sua vida cotidiana os requisitos da terapêutica, tanto a medicamentosa quanto a não medicamentosa.

Entende-se que a "Adesão ao tratamento antihipertensivo" pode ser influenciada por três grupos de fatores antecedentes os quais, atuando de modo inter-relacionado, podem determinar diferentes graus de adesão: os relativos ao próprio paciente, como as variáveis sociodemográficas, os conhecimentos e crenças que os pacientes têm sobre a doença e o tratamento, e o apoio da família; os relacionados à terapêutica farmacológica e não farmacológica; e os fatores relacionados ao sistema de saúde, entre os quais foram ressaltados a estrutura dos serviços de saúde e o processo de atendimento do portador de hipertensão.

Os fatores relacionados ao sistema de saúde, de modo especial, merecem ser analisados em profundidade em estudo de campo pois, conforme visto, a satisfação do hipertenso com o atendimento é fator primordial para sua adesão à terapêutica recomendada. Dentro desse contexto, a forma como os profissionais de saúde se relacionam com os pacientes hipertensos é um ponto chave para a "Adesão ao tratamento anti-hipertensivo".

As conseqüências da "Adesão ao tratamento anti-hipertensivo", como se procura evidenciar no modelo, são os resultados positivos que se pretende alcançar: uma pressão arterial controlada; a redução na incidência ou o retardamento na ocorrência de 
ARAÚJO, G. B. S.; GARCIA, T. R. Adesão ao tratamento anti-hipertensivo: uma análise conceitual. Revista Eletrônica de Enfermagem, v. 08, n. 02, p. 259 - 272, 2006. Disponível em http://www.fen.ufg.br/revista/revista8 2/v8n2a11.htm

possíveis complicações e a melhoria na qualidade de vida dos hipertensos.

Acredita-se que os resultados positivos servem de reforço para que os pacientes hipertensos mantenham o comportamento de adesão. Conforme já afirmado, essas conseqüências estão intimamente relacionadas, pois a adesão promove o controle dos níveis pressóricos que, por sua vez, resulta em redução na incidência ou retardamento na ocorrência de complicações e, conseqüentemente, na melhoria da qualidade de vida do hipertenso.

Além de reforçarem o comportamento de adesão, os resultados positivos, como se mostra na Fig. 1, retornam ao input do processo através da alça de realimentação, para que sejam feitos os ajustes necessários nos fatores que influenciam o fenômeno. Caso as conseqüências desejadas não sejam alcançadas, e comprovando-se a não adesão, esses ajustes se tornam ainda mais imprescindíveis. Nesse caso, é preciso verificar quais eventos antecedentes estão contribuindo para a não ocorrência do fenômeno, ou seja, deve-se buscar identificar que fatores, relativos ao paciente, à terapêutica ou ao sistema de saúde, estão contribuindo para a não adesão. A partir dessa identificação, deverão ser implementadas estratégias direcionadas a eliminar esses fatores ou a minimizar sua influência.

\section{CONSIDERAÇÕES FINAIS}

Levando em consideração os resultados da análise conceitual empreendida, como também tentando compreender a questão da "Adesão ao tratamento anti-hipertensivo" sob a ótica do modelo teórico elaborado, o estudo aponta para algumas implicações, tanto no que se refere à prática assistencial como à pesquisa.

Tomando como base os diversos eventos antecedentes necessários para a ocorrência da adesão, percebe-se a necessidade de considerar esse conceito como sendo multidimensional, pois envolve diferentes aspectos. Embora se deva considerar o portador de hipertensão como o foco central do processo, a ocorrência da adesão não depende unicamente dele, mas do conjunto de elementos constituintes do processo, ou seja, do conjunto portador de hipertensão $\leftrightarrow$ profissional de saúde $\leftrightarrow$ sistema de saúde. O esforço desenvolvido por um elemento isolado desse conjunto certamente não conduzirá a bons resultados, sendo necessária a ação conjunta para que a "Adesão ao tratamento antihipertensivo" seja alcançada. Vista a partir dessa perspectiva, não se reduz a complexidade da adesão ao âmbito individual, como muitas vezes ocorre na nossa prática diária.

No que se refere aos atributos do conceito analisado, sua identificação permitiu uma melhor clarificação e uma compreensão mais ampla do fenômeno. Os profissionais que atuam junto à clientela de hipertensos devem estar atentos a todos os aspectos do plano terapêutico, compreendendo que o esquema medicamentoso, embora importante, não garante por si só o sucesso do tratamento.

Por outro lado, faz-se necessário compreender que a adesão total ao plano terapêutico pode parecer uma tarefa hercúlea, irrealizável para muitos hipertensos. Ás vezes, o portador de hipertensão pode estar determinado a seguir o plano terapêutico e não dispor de condições econômicas para tal. Outras vezes, a estrutura e funcionamento dos serviços de saúde, ou o modo como o portador de hipertensão é visto e tratado pelos profissionais de saúde são os obstáculos a serem removidos, para facilitar a "Adesão ao tratamento anti-hipertensivo".

Hoje, o estudo tem a limitação de ter utilizado artigos publicados em periódicos indexados entre janeiro de 1995 e julho de 2001. Entretanto, os elementos identificados nesses artigos e incorporados ao modelo teórico do processo de "Adesão ao tratamento anti-hipertensivo" que foi construído permanecem atuais e, portanto, válidos para a compreensão do conceito. A identificação dos fatores antecedentes, dos atributos e das conseqüências mais freqüentemente associados ao conceito, propiciou subsídios para elaboração futura de instrumentos a serem utilizados em trabalhos de pesquisa relacionados à temática e para que se possa avaliar, em situações concretas da prática assistencial, a adesão dos pacientes ao tratamento anti-hipertensivo.

\section{REFERÊNCIAS BIBLIOGRÁFICAS}

AKASHI, D. et al. Tratamento anti-hipertensivo. Prescrição e custo de medicamentos - Pesquisa em hospital terciário. Arq. Bras. Cardiol., v.71, n.1, p.5577, 1998.

ALAVARCE, D. C.; PIERIN, A. M. G.; MION JR., D. A pressão arterial está sendo medida? Rev. Esc. Enf. USP, v. 34, n.1, p.84-90, 2000.

ARAÚJO, T. L. et al. Reflexo da hipertensão arterial no sistema familiar. Rev. Soc. Card. Estado de São Paulo, v. 8, n.2 (Supl A) p.1-6, 1998.

BITTAR, N. Maintaining long-term control of blood pressure: the role of improved compliance. Clinical Cardiology, v.18, n.6, p.12-16, 1995.

BRANDÃ̃O, A. P. et al. Curso de reciclagem em Cardiologia. Fascículo II. São Paulo: Pfeser, 1993.

BRASIL. Ministério da Saúde. Secretária de Políticas de Saúde, Cadernos de Atenção Básica, Hipertensão arterial sistêmica e Diabetes mellitus, Protocolo. Brasília, D.F., 2001.

CADE, N. V. O trabalho desenvolvido pelo enfermeiro: expectativas e percepção de hipertensos em tratamento ambulatorial. Rev. Enf. UERJ, v.7, n.2, p.148-153, 1999.

CAMPOS, E. P. Contribuição da psicologia ao tratamento do hipertenso. Folha Méd., v.113, n.2, p.153-156, 1996. 
ARAÚJO, G. B. S.; GARCIA, T. R. Adesão ao tratamento anti-hipertensivo: uma análise conceitual. Revista Eletrônica de Enfermagem, v. 08, n. 02, p. 259 - 272, 2006. Disponível em http://www.fen.ufg.br/revista/revista8 2/v8n2a11.htm

CAR, M. R.; PIERIN, A. M. G.; AQUINO, V. L. A. Estudo sobre a influência do processo educativo no controle da hipertensão arterial. Rev. ESC. Enf. USP., v.25, n.3, p.259-269, 1991.

CARVALHO FILHO, E. T.; CURIATI, J. A. E. Hipertensão arterial sistólica isolada no idoso. Rev. Bras. Med., v.53, n.10, p.989-998, 1996.

CARVALHO, F.; TELAROLLI JR., R.; MACHADO, J. C. M. S. Uma investigação antropológica na terceira idade: concepções sobre a hipertensão arterial. Cad. Saúde Pública, v.14, n.3, p.617-621, 1998.

CASTRO, V. D.; CAR, M. R. Dificuldades e facilidades dos doentes no seguimento do tratamento da hipertensão arterial. Rev. Esc. Enf. USP, v.33, n.33, p.294-304, 1999.

CASTRO, V. D.; CAR, M. R. O cotidiano da vida de hipertensos: mudanças, restrições e reações. Rev. ESC. Enf. USP, v.34, n.2, p.145-153, 2000.

CHOR, D. Hipertensão arterial entre funcionários de banco estatal no Rio de Janeiro. Hábitos de vida e tratamento. Arq. Bras. Cardiol., v.71, n.5, p.100-119, 1998.

CLARK, M. J.; CURRAN, C.; NOJI, A. The effects of community health nurse monitoring on hypertension identification and control. Public Health Nursing, v.17, n.6, p.452-459, 2000.

COSTA, A. Hipertensão arterial sistêmica como fator de risco. Compacta - Temas em cardiologia, v.3, n.2, p.5-10, 2001.

COSTA, S. A. Prevenção e controle da hipertensão arterial na aeronáutica - um programa viável. Rev. Med. Aeronaut. Bras., v.48, p.19-23, 1998.

CRUZ, I. C. F. et al. O cliente/família com hipertensão arterial essencial: considerações sobre o diagnóstico e tratamento de enfermagem na consulta. Rev. Enf. UERJ, v.3, n.1, p.71-76, 1995.

CRUZ, I. C. F.; LIMA, R. Detecção dos fatores de risco para a hipertensão arterial na equipe de enfermagem. Rev. Enf. UERJ, v.6, n.1, p.223-232, 1998.

CURY JR., A. J. Hipertensão arterial e qualidade de vida. Âmbito Hospitalar, v.58, n.8, p.58-60, 1996.

DEVINE, E. C.; REIFSCHNEIDER, E. A metaanalysis of the effects of psycho educational care in adults of hypertension. Nursing Research, v.44, n.4, 1995.

FEITOSA, G. S. Tratamento da hipertensão arterial no negro. Hiper Ativo, v.3, n.3, p.180-185, 1996.

FISCHMAN, T. The 90 - second intervention: a patient compliance mediated technique to improve and control hypertension. Public Health Reports, v.110, n.2, p.173-178, 1995.

FREITAS, O. C. et al. Prevalence of hypertension in the urban population of Catanduva, in the state of São Paulo, Brazil. Arq. Bras. Cardiol., v.77, n.1, p.16-21, 2001.

GRUENINGER, U. J. Arterial hypertension: lessons from patient education. Patient Education and Counseling, v. 26, p.37-55, 1995.
GUERRA-RICCIO, G. M. Adesão do paciente hipertenso ao tratamento: influência da freqüência do atendimento. São Paulo, 2001, 122 p. Tese (Doutorado) - Faculdade de Medicina, Universidade de São Paulo.

HUNGERBUHLER, $P$. et al. Compliance with medication among out patients with uncontrolled hypertension in the Seychelles. Bulletin of the Word Health Organization, v.73, n.4, p.437-442, 1995.

JARDIM, P. C. B. V.; SOUZA, A. L. L.; MONEGO, E. T. Atendimento multiprofissional ao paciente hipertenso. Medicina, v.29, p.232-238, 1996.

KAPLAN, N. M. Resistant hypertension: what to do after trying "the usual". Geriatrics, v.50, n.5, p.2438,1995 .

KJELLGREN, K. I. et al. Antihypertensive treatment and patient autonomy - the follow-up appointment as a resource for care. Patient Education and Counseling, v.40, p.39-49, 2000.

KJELLGREN, K. I.; AHLNER, J.; SÄLJÖ, R. Taking antihypertensive medication - controlling or cooperating with patients? International Journal of Cardiology, v.47, p.257-268, 1995.

KUNCL, N.; NELSON, K. M. Antihypertensive drugs balancing risks and benefits. Nursing, p.46-49, 1997.

KYNGÄS, H.; LAHDENPERÄ, T. Compliance of patients with hypertension and associated factors. Journal of Advanced Nursing, v.29, n.4, p.832-839, 1999.

LAHDENPERÄ, T. S.; KYNGÄS, H. A. Compliance and its evaluation in patients with hypertension. Journal of Clinical Nursing, v.9, p.826-833, 2000.

LESSA, I. Estudos Brasileiros sobre a epidemiologia da Hipertensão Arterial: análise crítica dos estudos de prevalência. Informe epidemiológico do SUS, Brasília, v.2, n.3, p.59-75, 1993.

MARCON, S. S. et al. Comportamento preventivo de servidores da UEM hipertensos e a aderência ao programa de assistência ao hipertenso do ambulatório. Ciencia Y Enfermería, v.1, n.1, p.33-42, 1995.

MEDEL, E. S. Adherencia al control de los pacientes hipertensos y Factores que la Influencian. Ciência $y$ Enfermeria, v.3, p.49-58, 1997.

MION JR. et al. Conhecimento, preferências e perfil dos hipertensos quanto ao tratamento farmacológico e não farmacológico. J. Bras. Nefrol., v.17, n.4, p.229-236, 1995.

MOREIRA, T. M. M.; MACIEL, I. C. F.; ARAÚJO, T. L. Trabalhando a auto-ajuda em grupo no controle da hipertensão. Nursing, p.20-24, 1999.

PEPPER, G. A. Pharmacology of antihypertensive drugs, JOGNN, v.28, p.649-659, 1999.

POVOA, R. Tratamento da hipertensão arterial sistêmica. Compacta - Temas em cardiologia. v.3, n.2, p.5-10, 2001.

ROUQUAYROL, M. Z. Distribuição das doenças e dos agravos à saúde coletiva. In: ROUQUAYROL, M. Z. Epidemiologia e saúde. 4ed. Rio de janeiro: MEDSI, 1994. Cap.4, p.77-155. 
RUDD, P. Clinicians and patients with hypertension: unsettled issues about compliance. American Heart Journal, v. 130, n.3, p.572-579, 1995.

SALA, A.; NEMES FILHO, A.; ELUF-NETO, J. Avaliação da efetividade do controle da hipertensão arterial em unidade básica de saúde. Rev. Saúde Pública, v.30, n.2, p.161-167, 1996.

SARQUIS, L. M. M. et al. A adesão ao tratamento na hipertensão arterial: análise da produção científica. Rev. Esc. Enf. USP, v.32, n.4, p.335-353, 1998.

SOCIEDADE BRASILEIRA DE HIPERTENSÃO SBH. 31/7/2003 - OMS alerta para baixa adesão ao tratamento. [online]. Disponível em: http://www.sbh.org.br/noticias.asp?codigo $=149$

[Acesso em 30 set. 2006].

SMELTZER, S. C.; BARE, B. C. Brunner e Suddarth. Tratado de enfermagem médico-cirúrgica. 8. ed. Rio de Janeiro: Guanabara Koogan, 1998.

SVENSSON, S. et al. Reasons for adherence with antihypertensive medication. International Journal of Cardiology, v.76, p.157-163, 2000.

TRENTINI, M.; TOMASI, N.; POLAK, Y. Prática educativa na promoção da saúde com grupo de pessoas hipertensas. Cogitare Enferm., v.1, n.2, p.19-24, 1996.

VIJAYALAKSMI, T.; RAZIA, R.; PRAKASAMMA, M. Knowledge and learning needs of clients with hypertension. The Nursing Journal of Índia, v.87, n.4, p.74-76, 1997.

VIVIAN, B. G. Reconceptualizing compliance in home health care. Nursing Forum, v.31, n.2, p.5-13, 1996.

WALKER, L. O.; AVANT, K. C. Strategies for theory Construction in Nursing. 3. ed. Norwaek, CT, Appleton e Lange, 1995.

WANG, C.; ABBOTT, J. Development of a community-based diabetes and hypertension preventive program. Public Health Nursing, v.15, n.6, p.406-414, 1998.

WILLIS, J. Patient compliance. Nursing, v.96, n.35, p.36-37, 2000. 